\title{
Deep learning model for detection of hypoalbuminemia using electrocardiography
}

Short title: Artificial intelligence for hypoalbuminemia

Joon-myoung Kwon, MD MS ${ }^{\mathrm{a}, \mathrm{b}, \mathrm{c}, \mathrm{d} *}$, Soo Youn Lee, MD MS ${ }^{\mathrm{b}, \mathrm{e} *}$, Yoon-Ji Lee, BS ${ }^{\mathrm{a} *}$, Yong-Yeon Jo PhD ${ }^{\mathrm{a}}$, Min-Seung Jung, BS ${ }^{\mathrm{a}}$, Yong-Hyeon Cho, BS ${ }^{\mathrm{a}}$, Jae-Hyun Shin, BS ${ }^{\mathrm{a}}$, Jang-Hyeon Ban, MS ${ }^{\mathrm{d}}$, Kyung-Hee Kim, MD PhD ${ }^{\text {bee }}$, Jinsik Park, MD PhD ${ }^{\mathrm{e}}$, and Byung-Hee Oh, MD, $\mathrm{PhD}^{\mathrm{e}}$

${ }^{a}$ Medical research team, Medical AI, co. Seoul, South Korea; ${ }^{b}$ Artificial Intelligence and Big Data Research Center, Sejong Medical Research Institute, Bucheon, South Korea; ${ }^{c}$ Department of Critical Care and Emergency Medicine, Mediplex Sejong Hospital, Incheon, South Korea; ${ }^{\mathrm{d}}$ Medical R\&D center, Body friend, co. Seoul, South Korea; ${ }^{\mathrm{e} D i v i s i o n ~ o f ~}$ Cardiology Cardiovascular Center, Mediplex Sejong Hospital, Incheon, South Korea; All authors take responsibility for all aspects of the reliability and freedom from bias of the data presented and their discussed interpretation.

*These three authors contributed equally to this work.

Word count of text: 2957

Corresponding Author:

Joon-myoung Kwon, MD, MS

Department of Emergency Medicine, Mediplex Sejong Hospital, Incheon, South Korea; Artificial Intelligence and Big Data Research Center, Sejong Medical Research Institute, Bucheon, South Korea

20, Gyeyangmunhwa-ro, Gyeyang-gu, Incheon, Republic of Korea

Tel: 82-32-240-8129; Fax: 82-32-240-8094; E-mail: kwonjm@sejongh.co.kr

Soo Youn Lee, MD, MS

Division of Cardiology, Department of Internal Medicine, Cardiovascular Center, Mediplex Sejong Hospital, Incheon, Korea

20, Gyeyangmunhwa-ro, Gyeyang-gu, Incheon, Republic of Korea

Tel: 82-32-240-8568; Fax: 82-32-240-8094; E-mail: leesy@sejongh.co.kr 


\section{Abstract}

Background: Albumin has a pivotal role in the homeostasis of osmotic pressure and is associated with cardiovascular, nephrotic, hepatic, and nutritional diseases. The detec tion of hypoalbuminemia is a cornerstone for diagnosis of hidden diseases and patient deterioration. We developed and validated a deep-learning-based model (DLM) for det ection of hypoalbuminemia using electrocardiography (ECG).

Methods: This historical cohort study included data from consecutive patients from tw o hospitals. The patient data in one hospital were divided into development $(82,499 \mathrm{E}$ CGs from 54,248 patients) and internal validation (20,664 ECGs from 20,664 patients) datasets, whereas the patient data in the other hospital were included in only an exte rnal validation (37,421 ECGs from 37,421 patients) dataset. An DLM was developed using a 12-lead ECG signal, age, and sex from the development dataset. The endpoint was hypoalbuminemia, defined by serum albumin concentration below $3.5 \mathrm{~g} / \mathrm{dL}$.

Results: During the internal and external validations, the areas under the receiver ope rating characteristic curve of the DLM for the detection of hypoalbuminemia were 0.8 $87(0.877-0.897)$ and $0.888(0.880-0.896)$, respectively. Among the 27,400 individuals without hypoalbuminemia at the initial laboratory exam, those identified by the DLM as higher-risk patients had a significantly larger change in developing hypoalbuminemi a than those in the low-risk group $(7.09 \%$ vs. $1.01 \%, p<0.001)$ during 24 months. The sensitivity map showed that the DLM focused on the T wave and QRS complex for the detection of hypoalbuminemia.

Conclusions: The DLM exhibited a high accuracy for hypoalbuminemia detection and prediction using 12-, 6-, and single-lead ECGs.

Keywords: Electrocardiography; Albumins; Deep Learning; Artificial Intelligence 


\section{Introduction}

As albumin provides $80 \%$ of the total colloid osmotic pressure of plasma and $50 \%$ of protein content, it has a pivotal role in the maintenance of homeostasis.[1] $\mathrm{Se}$ rum albumin transports several different fat-soluble hormones and drugs and serves as a plasma buffer, maintaining physiological $\mathrm{pH}$ levels, and antioxidant, involved in the scavenging of oxygen free radicals.[2,3] Hypoalbuminemia is common in numerous diseases, including liver cirrhosis, malnutrition, nephrotic syndrome, heart failure, and $\mathrm{s}$ epsis.[4,5] The evaluation of the albumin level is a cornerstone for diagnosis and proper treatment. The monitoring of the albumin level is crucial for patients who have diseases that impair the retention and excretion of fluid, such as heart failure and liver cirrhosis, and patients who take medications transported by albumin, such as diuretics.[6-8]

As the symptom of hypoalbuminemia is nonspecific, it is challenging to diagnose it with only the medical history and physical examinations until the condition is uncompensated and complications occur.[9] The gold standard for diagnosing hypoalbuminemia is a laboratory examination to measure the concentration of albumin. However, the laboratory tests are invasive, costly, and require specialized equipment and infrastructure, such as trained medical staff for sampling blood and hematology analysis devices for assessment with biochemical reagents. Detecting hypoalbuminemia in daily life is important to monitor the health status and detect deteriorating events. However, the evaluation using a laboratory exam could not be used for this purpose. Moreover, laboratory tests are too expensive to use in low-income countries for screening hypoalbuminemia.

Albumin represents a circulating endogenous reservoir of nitric oxide (NO) and acts as a donor for $\mathrm{NO}$, which has diverse cardiovascular effects.[10] Albumin is associated with right ventricular free wall longitudinal strain and development and prognosis of cardiovascular diseases, which can be correlated with electrocardiography (ECG).[11-16] 
However, it is not simple to provide diagnostic tools using such subtle ECG changes based on conventional statistical methods. Recent studies have shown that deep learning models using ECG could detect diverse diseases, including anemia, valvular heart disease, heart failure, myocardial infarction, and electrolyte imbalances.[17-25] In this study, we developed and validated a deep-learning-based model (DLM) to detect hypoalbuminemia using ECG.

\section{Methods}

\section{Study design and population}

We carried out a retrospective multi-center diagnostic study in which a DLM was developed using ECGs, and then internally and externally validated. We excluded individuals with missing demographic, electrocardiographic, and albumin laboratory exam information. Data from the Sejong General Hospital (SGH) were used for the development and internal validation. In the SGH, we identified patients with at least one standard digital 10-s 12-lead ECG acquired in the supine position within the study period (October 1, 2016 to August 31, 2020) and at least one laboratory exam for albumin within 30 min after the index ECG. The individuals who visited the general health checkup, outpatient department, and emergency department and were admitted to SGH were the study population for the development and internal validation datasets. As shown in Supplementary material 1, patients who underwent a follow-up albumin laboratory examination after an initial evaluation were assigned $\mathrm{t}$ o an internal validation dataset. Patients who had not underwent follow-up laboratory exam were assigned to a development dataset used to develop the DLM. Subsequently , we evaluated the accuracy of the DLM using the internal validation dataset. Data from Mediplex Sejong Hospital (MSH) were used for an external validation. We identified the patients who were admitted to MSH during the study period (March 1, 2017 to August 31, 2020) and who had at least one ECG and one albumin laboratory exam within 30 min after 
the index ECG. As the purpose of the validation data was to assess the accuracy of the DLM, we used only one ECG from each patient for the internal and external validation datasets, the time-closest to the albumin laboratory exam in the study period.

This study was approved by the institutional review boards of SGH and MSH.

Clinical data, including digitally stored ECGs, albumin laboratory exam values, age, and sex, were obtained from both hospitals. Both institutional review boards waived the need for informed consent because of the retrospective nature of the study using fully anonymized ECG and health data and minimal harm.

\section{Procedures}

The predictor variables were ECG, age, and sex. Digitally stored 12-lead ECG data, 5000 numbers for each lead, were recorded over $10 \mathrm{~s}(500 \mathrm{~Hz})$. We removed 1-s intervals at both beginning and end of each ECG because of the more artifacts than in other parts. Thus, the length of each ECG was $8 \mathrm{~s}$ (4000 numbers). We created a dataset using the entire 12-lead ECG data. We also used partial datasets from the 12-lead ECG data, such as six-limb-lead and single-lead datasets (lead I). We selected the sets of leads because the data can easily be recorded by wearable and pad devices in contact with the hands and legs. Consequently, when we developed and validated the DLM using 12-lead ECGs, we used a dataset of twodimensional (2D) data of $12 \times 4000$ numbers. When we developed and validated an algorithm using six-lead ECGs, we used datasets of $6 \times 4000$ numbers, while for the singlelead ECGs, we used datasets of $1 \times 4000$ numbers. The endpoint of this research was hypoalbuminemia, defined by serum albumin concentration $<3.5 \mathrm{~g} / \mathrm{dL}$.

As shown in Supplementary material 2, the DLM was developed using several hidden layers of neurons to learn complex hierarchical nonlinear representations from the data. A residual block with five stages included two convolution layers, two batch normalizations, one max-pooling, and one dropout layer (repeated), as shown in Supplementary material 2. We used 
$1 \times 4$ max-pooling layers between blocks 1 and 4 and $2 \times 4$ max-pooling layers between blocks 4 and 5. The last convolutional layer of the residual block was connected to a flattened layer, which was fully connected to a one-dimensional (1D) layer composed of 128 nodes. The input layer of epidemiology data (age and sex) was concatenated with the 1D layer. Two fully connected 1D layers were connected to the output node, which was composed of one node. The output node used a softmax function as an activation function because the output of the softmax function was between 0 and 1 . The architecture of the DLM was evaluated and verified using a grid search. We developed an additional DLM using six-limb-lead and single-lead ECGs.

\section{Statistical analysis}

Continuous variables are presented as mean values (standard deviations (SDs)) and compared using the unpaired Student's $t$-test or Mann-Whitney $U$ test. Categorical variables are expressed as frequencies and percentages and compared using the $\chi^{2}$ test. At each input (ECG) of validation data, the DLM calculated the possibility of a primary endpoint in the range of 0 (non-hypoalbuminemia) to 1 (hypoalbuminemia). To verify the DLM performance, we compared the probability calculated by the DLM with the presence of a hypoalbuminemia in the internal and external validation datasets. To this end, we used the area under the receiver operating characteristic curve (AUC). We applied the cutoff point to the internal and external validation data to calculate the sensitivity, specificity, positive predictive value, and negative predictive value, which were confirmed at the operating point by Youden J statistics in the development data.[26] The 95\% confidence intervals (CIs) were used for all measures of diagnostic performances, except for AUC. The CIs for AUC were determined based on the Sun-Su optimization of the De-long method using the pROC package in R (The R Foundation for Statistical Computing, Vienna, Austria). A significant difference in patient characteristics was defined by two-sided $p<0.001$. Statistical analyses were carried out using the R software, version 3.4. In addition, we used the PyTorch's open- 
source software library as the backend and Python (version 3.6) for the analysis.

\section{Visualization of the developed XDM for interpretation}

To understand the developed model and compare it to existing medical knowledge, it was necessary to identify a region that had a significant effect on the decision of the developed DLM. We employed a sensitivity map using a saliency method.[27,28] The map was computed using the first-order gradients of the classifier probabilities with respect to the input signals. If the probability of the classifier was sensitive to a specific region of the signal, the region would be considered significant in the model. In other words, we verified the region of the ECG that was associated with hypoalbuminemia using a sensitivity map. We used a gradient class activation map as a sensitivity map and guided the gradient backpropagation method. We verified the variable importance values of ECG features, age, and sex in logistic regression, random forest, and deep learning using the deviance difference, mean decreased Gini, and relative importance based on the Garson's algorithm, respectively.[29]

\section{Verification of the DLM performance to predict the hypoalbuminemia by a subgroup analysis}

We hypothesized that the ECGs would display subtle abnormal patterns in the prehypoalbuminemia phase and that the developed DLM would classify certain cases as abnormal, yielding a false positive (a study subject classified as having hypoalbuminemia but considered as non-hypoalbuminemia) as the initial result. We carried out a subgroup analysis of patients who underwent follow-up laboratory examinations in the internal and external validation datasets. The difference in data between the initial and follow-up echocardiography data was over $14 \mathrm{~d}$. Among those patients, we verified the development of hypoalbuminemia in patients who were initially considered non-hypoalbuminemia patients, whose serum albumin concentration was $3.5 \mathrm{~g} / \mathrm{dL}$ or higher. The DLM data were categorized 
into high- and low-risk groups based on the risk score using cutoff values, which were determined using the Youden's J statistic with the development dataset.[26] We used the Kaplan-Meier method to analyze the hypoalbuminemia development over 24 months.

\section{Results}

The eligible population included 74,919 and 37,426 patients from SGH and MSH, respectively. We excluded 7 and 5 patients from SGH and MSH, respectively, because of missing clinical information (age and sex), laboratory albumin information, or ECG data (Supplementary material 1). The study included a total of 112,333 patients, of whom 3,812 had hypoalbuminemia. The DLM was developed using a development dataset of 82,499 ECGs of 54,248 patients from SGH. The performance of the algorithm was then verified using 20,664 ECGs of 20,664 patients from SGH in the internal validation dataset and 37,421 ECGs of 37,421 patients from MSH in the external validation dataset. In the case of hypoalbuminemia, the ECG had a prolonged QRS duration, rightward T-wave axis, and tachycardia (Table 1).

During the internal and external validations, the AUCs of the DLM for detecting hypoalbuminemia as the endpoint using 12-lead ECGs were 0.887 (95\% confidence interval, $0.877-0.897)$ and $0.888(0.880-0.896)$, respectively (Figure 1). The AUCs of the DLM for detecting hypoalbuminemia using six-lead ECGs during the internal and external validations were $0.871(0.859-0.882)$ and $0.880(0.871-0.889)$, respectively. The AUCs of the DLM using single-lead ECGs during the internal and external validations were $0.854(0.842-0.867)$ and $0.879(0.870-0.887)$, respectively (Figure 1).

The DLM described the important ECG region for hypoalbuminemia detection. As shown in Figure 2, the DLM focused on the QRS complex and T wave for the detection of hypoalbuminemia. As shown in Supplementary material 3, the variable importance diffe 
red for each machine learning model. The random forest used the T-wave axis, while the DLM used the QT interval as an important predictive variable.

Our study comprised 28,482 patients $(20,664$ and 7,818 patients in the internal and external validation datasets, respectively) with follow-up laboratory results. Amon $\mathrm{g}$ them, 27,400 patients were normal (non-hypoalbuminemia) at the initial laboratory e xamination. We carried out a subgroup analysis of hypoalbuminemia development after the initial laboratory examination for the 27,400 patients, of whom 869 developed hy poalbuminemia within 24 months. The high-risk group of the DLM exhibited a signifi cantly higher hazard (Figure 3) and higher development rate of hypoalbuminemia than those of the low-risk group $(7.09 \%$ vs. $1.01 \%, p<0.001)$.

\section{Discussion}

We developed and validated the DLM based on an ensemble network for hypoalbuminemia detection using 12-, six-, and single-lead ECGs and demonstrated a reasonable performance. Subsequently, we visualized our DLM to determine the regions and characteristics of the ECG that were used for hypoalbuminemia detection and verified the important variable for the decision in diverse statistical methods, such as logistic regression, random forest, and DLM. We carried out a subgroup analysis for non-hypoalbuminemia (normal) patients at the initial laboratory examination. The DLM could predict the development of hypoalbuminemia. To the best of our knowledge, this study is the first that develops a DLM for detection and prediction of hypoalbuminemia using ECG and demonstrates interpretable patterns of decision making using the DLM.

The development of a reliable tool for detection and prediction of hypoalbuminemia is the cornerstone for the monitoring of the albumin status and early management to prevent an irreversible disease progression.[6-8] Hypoalbuminemia is also associated with the 
nutritional status of patients. As one of the pathophysiologies of hypoalbuminemia is associated with an increase in capillary permeability and altered kinetics of serum albumin in inflammatory states, hypoalbuminemia is a reflection of the extent of physiologic stress from disease- and trauma-related inflammations.[30] Hypoalbuminemia is associated with liver diseases, kidney diseases, and malnutrition or malabsorption based on albumin synthesis, loss, and intake, respectively.[4,5] Therefore, the detection of hypoalbuminemia is important to not only monitor nutritional and homeostasis general conditions, but also early-diagnose other diverse diseases using hypoalbuminemia as a surrogate factor and detect deterioration of the patient.[11-16]

Although laboratory tests for albumin are diagnostic tests for hypoalbuminemia, they require blood sampling and infrastructure for a blood analysis. Therefore, the detection of hypoalbuminemia based on laboratory tests could not be used in daily life and low-income countries. As stated above, albumin is an endogenous reservoir of NO and acts as a donor for NO.[10] The cardioprotective roles of NO include regulation of blood pressure and vascular tone, inhibition of platelet aggregation and leukocyte adhesion, and prevention of smooth muscle cell proliferation. Reduced bioavailability of NO is considered one of the central factors common in endothelial dysfunction. The underlying pathology for most cardiovascular diseases is atherosclerosis, which is associated with endothelial dysfunction.[10] The albumin level has been significantly negatively correlated with right ventricular free wall longitudinal strain.[11] Albumin is a strong prognostic factor for cardiovascular diseases. As the albumin level affects diverse cardiovascular statuses, we hypothesized that we could detect hypoalbuminemia based on ECG.[11-16]

The most important aspect of deep learning is its ability to extract features and develop an algorithm using various types of data, such as images, 2D data, and waveforms.[31] Attia et al. and our study group developed a DLM to screen for heart failure, arrhythmia, valvular 
heart disease, left ventricular hypertrophy, electrolyte imbalance, and anemia.[17-25] Deep learning is criticized for its unreliable outcomes because of the low transparency of the process, so-called black box. Therefore, we used a sensitivity map to describe the abnormal findings that affected the decision of the DLM for the detection of RI and description of the variable importance of ECG features. Using this method, we verified the ECG region and features associated with hypoalbuminemia. Conventional methods are based on hypotheses of researchers. For deep learning methods, such as the DLM and sensitivity mapping in this study, the findings are not based on previous medical knowledge of humans, but on data. Therefore, we could provide new knowledge only by the data, without human prejudice. Deep learning could discover the complex hierarchical nonlinear representation that could not be discovered using conventional statistical methods, such as logistic regression. In this study, we verified the important ECG region for the detection of hypoalbuminemia from waveform data. We verified that hypoalbuminemia could be detected and predicted using ECG based on the DLM. The sensitivity map and variable importance information showed that QRS complex, T wave, QT interval, and PR interval were correlated with the detection of hypoalbuminemia. These findings are in agreement with the results of previous studies. Heaf et al. and Madias et al. showed that the amplitude of the QRS complex was associated with albumin levels.[32,33] Toma et al. and $\mathrm{Wu}$ et al. showed that prolonged QT intervals and $\mathrm{T}$ accentuated deceleration of the T wave correlated with hypoalbuminemia.[13,34]

In this study, the DLM could predict the development of hypoalbuminemia. The DLM may detect structural and physiological changes that affect the vulnerability of hypoalbuminemia. These findings suggest that the DLM could be used to not only diagnose hypoalbuminemia but also screen patients who have characteristics of hypoalbuminemia risk. The reliable performances of the six- and single-lead ECG-based DLMs indicate that hypoalbuminemia could be screened with both conventional 12-lead ECG and life-type or 
wearable ECG device or simple monitoring device. Using this algorithm, developing countries with limited medical resources could be screened with simple devices. In developed countries, patients could be monitored using diverse life-type devices and could be alerted regarding disease progression in daily life.

This study has several limitations. First, we validated the DLM using retrospective da ta. However, it is necessary to validate the DLM with prospective studies and data from daily life. Studies related to the clinical significance of the new technology are required for applicat ion in clinical practice. In our next study, we will verify the DLM performance and significan ce with a prospective study in daily clinical practice. Second, this study was carried out only i $\mathrm{n}$ two hospitals in Korea. Hence, it is necessary to validate the DLM with patients in other co untries. Third, the decision process of the algorithm must be further investigated based on deep learning. For example, additional experiments must be performed to understand the d eep learning process and determine the characteristics of the QRS complex that affect the DLM's decision. This will be investigated in our next study.

\section{Conclusion}

The DLM exhibited high performances and accuracy in the detection of hypoalbuminemia using ECG and successfully predicted the development of hypoalbuminemia.

Acknowledgments: This research was results of a study on the "AI voucher" Project, supported by the 'Ministry of Science and ICT and National IT Industry Promotion Agency of South Korea.

Sources of Funding: This work was supported by the National Research Foundation of Korea (NRF) grant funded by the Korea government(MSIT) (No. 2020R1F1A1073791) 
Disclosures: SYL, KHK, and BHO declare that they have no competing interests. JK and JP are co-founder and YYJ, MSJ, YJL, YHC, and JHS are researchers of Medical AI Co., a medical artificial intelligence company. JK and JHB are researchers of Body friend Co. There are no products in development or marketed products to declare. This does not alter our adherence to this journal.

Affiliations: JK, SYL, KHK, JP, and BHO (Mediplex Sejong Hospital); JK, YJL, YYJ, MSJ, YHC, and JHS (Medical AI Co. Ltd.); JK and JHB (Bodyfriend Co. Ltd.)

Author contributions: YJL, YYJ and SYL performed data analysis and verified the clinical coding. MSJ, YHC, HJS, and JHB contributed to the study idea and design as well as data collection, performed data analysis, and contributed to subsequent drafts. KHK, JP, and BHO contributed to data collection and revised the manuscript. JK is the principal investigator and contributed to the study idea and design, data analysis, verified the clinical coding, and contributed to subsequent drafts.

Data availability statement: The data underlying this article will be shared on reasonable request to the corresponding author. 


\section{References}

[1] J. Boldt, RETRACTED: Use of albumin: an update, Br. J. Anaesth. 104 (2010) 276284. https://doi.org/10.1093/bja/aep393.

[2] M. Taverna, A.-L. Marie, J.-P. Mira, B. Guidet, Specific antioxidant properties of human serum albumin, Ann. Intensive Care. 3 (2013) 4. https://doi.org/10.1186/21105820-3-4.

[3] B. Elsadek, F. Kratz, Impact of albumin on drug delivery - New applications on the horizon, J. Control. Release. 157 (2012) 4-28.

https://doi.org/10.1016/j.jconrel.2011.09.069.

[4] S. Finfer, R. Bellomo, N. Boyce, J. French, J. Myburgh, R. Norton, SAFE Study Investigators, A comparison of albumin and saline for fluid resuscitation in the intensive care unit., N. Engl. J. Med. 350 (2004) 2247-56.

https://doi.org/10.1056/NEJMoa040232.

[5] P. Sort, M. Navasa, V. Arroyo, X. Aldeguer, R. Planas, L. Ruiz-del-Arbol, L. Castells, V. Vargas, G. Soriano, M. Guevara, P. Ginès, J. Rodés, Effect of Intravenous Albumin on Renal Impairment and Mortality in Patients with Cirrhosis and Spontaneous Bacterial Peritonitis, N. Engl. J. Med. 341 (1999) 403-409. https://doi.org/10.1056/NEJM199908053410603.

[6] R. Mehrotra, U. Duong, S. Jiwakanon, C.P. Kovesdy, J. Moran, J.D. Kopple, K. Kalantar-Zadeh, Serum Albumin as a Predictor of Mortality in Peritoneal Dialysis: Comparisons With Hemodialysis, Am. J. Kidney Dis. 58 (2011) 418-428. https://doi.org/10.1053/j.ajkd.2011.03.018.

[7] S. Uthamalingam, J. Kandala, M. Daley, E. Patvardhan, R. Capodilupo, S.A. Moore, J.L. Januzzi, Serum albumin and mortality in acutely decompensated heart failure, Am. Heart J. 160 (2010) 1149-1155. https://doi.org/10.1016/j.ahj.2010.09.004. 
[8] M. Duffy, S. Jain, N. Harrell, N. Kothari, A. Reddi, Albumin and Furosemide Combination for Management of Edema in Nephrotic Syndrome: A Review of Clinical Studies, Cells. 4 (2015) 622-630. https://doi.org/10.3390/cells4040622.

[9] A. Gatta, A. Verardo, M. Bolognesi, Hypoalbuminemia, Intern. Emerg. Med. 7 (2012) 193-199. https://doi.org/10.1007/s11739-012-0802-0.

[10] K. NASEEM, The role of nitric oxide in cardiovascular diseases, Mol. Aspects Med. 26 (2005) 33-65. https://doi.org/10.1016/j.mam.2004.09.003.

[11] J.A. Borovac, D. Glavas, Z. Susilovic Grabovac, D. Supe Domic, L. Stanisic, D. D’Amario, D. Duplancic, J. Bozic, Right Ventricular Free Wall Strain and Congestive Hepatopathy in Patients with Acute Worsening of Chronic Heart Failure: A CATSTAT-HF Echo Substudy, J. Clin. Med. 9 (2020) 1317. https://doi.org/10.3390/jcm9051317.

[12] A. Ronit, D.M. Kirkegaard-Klitbo, T.L. Dohlmann, J. Lundgren, C.A. Sabin, A.N. Phillips, B.G. Nordestgaard, S. Afzal, Plasma Albumin and Incident Cardiovascular Disease, Arterioscler. Thromb. Vasc. Biol. 40 (2020) 473-482. https://doi.org/10.1161/ATVBAHA.119.313681.

[13] C.-C. Wu, Y.-C. Lu, T.-H. Yu, C.-P. Wang, W.-C. Hung, W.-H. Tang, I.-T. Tsai, F.M. Chung, Y.-J. Lee, J.-J. Hu, Serum albumin level and abnormal corrected QT interval in patients with coronary artery disease and chronic kidney disease, Intern. Med. J. 48 (2018) 1242-1251. https://doi.org/10.1111/imj.13796.

[14] A. Ancion, S. Allepaerts, C. Oury, A.-S. Gori, L.A. Piérard, P. Lancellotti, Serum albumin level and hospital mortality in acute non-ischemic heart failure, ESC Hear. Fail. 4 (2017) 138-145. https://doi.org/10.1002/ehf2.12128.

[15] M. Cesari, B.W.J.H. Penninx, A.B. Newman, S.B. Kritchevsky, B.J. Nicklas, K. Sutton-Tyrrell, S.M. Rubin, J. Ding, E.M. Simonsick, T.B. Harris, M. Pahor, 
Inflammatory Markers and Onset of Cardiovascular Events, Circulation. 108 (2003) 2317-2322. https://doi.org/10.1161/01.CIR.0000097109.90783.FC.

[16] H. Bahrami, D.A. Bluemke, R. Kronmal, A.G. Bertoni, D.M. Lloyd-Jones, E. Shahar, M. Szklo, J.A.C. Lima, Novel Metabolic Risk Factors for Incident Heart Failure and Their Relationship With Obesity, J. Am. Coll. Cardiol. 51 (2008) 1775-1783. https://doi.org/10.1016/j.jacc.2007.12.048.

[17] Z.I. Attia, S. Kapa, F. Lopez-Jimenez, P.M. McKie, D.J. Ladewig, G. Satam, P.A. Pellikka, M. Enriquez-Sarano, P.A. Noseworthy, T.M. Munger, S.J. Asirvatham, C.G. Scott, R.E. Carter, P.A. Friedman, Screening for cardiac contractile dysfunction using an artificial intelligence-enabled electrocardiogram, Nat. Med. 25 (2019) 70-74. https://doi.org/10.1038/s41591-018-0240-2.

[18] Z.I. Attia, P.A. Friedman, P.A. Noseworthy, F. Lopez-Jimenez, D.J. Ladewig, G. Satam, P.A. Pellikka, T.M. Munger, S.J. Asirvatham, C.G. Scott, R.E. Carter, S. Kapa, Age and Sex Estimation Using Artificial Intelligence From Standard 12-Lead ECGs, Circ. Arrhythmia Electrophysiol. 12 (2019). https://doi.org/10.1161/CIRCEP.119.007284.

[19] C.D. Galloway, A. V. Valys, J.B. Shreibati, D.L. Treiman, F.L. Petterson, V.P. Gundotra, D.E. Albert, Z.I. Attia, R.E. Carter, S.J. Asirvatham, M.J. Ackerman, P.A. Noseworthy, J.J. Dillon, P.A. Friedman, Development and Validation of a DeepLearning Model to Screen for Hyperkalemia From the Electrocardiogram, JAMA Cardiol. 4 (2019) 428. https://doi.org/10.1001/jamacardio.2019.0640.

[20] Z.I. Attia, P.A. Noseworthy, F. Lopez-Jimenez, S.J. Asirvatham, A.J. Deshmukh, B.J. Gersh, R.E. Carter, X. Yao, A.A. Rabinstein, B.J. Erickson, S. Kapa, P.A. Friedman, An artificial intelligence-enabled ECG algorithm for the identification of patients with atrial fibrillation during sinus rhythm: a retrospective analysis of outcome prediction, 
Lancet. 394 (2019) 861-867. https://doi.org/10.1016/S0140-6736(19)31721-0.

[21] Y. Cho, J.-M. Kwon, K.-H. Kim, J.R. Medina-Inojosa, K.-H. Jeon, S. Cho, S.Y. Lee, J. Park, B.-H. Oh, Artificial intelligence algorithm for detecting myocardial infarction using six-lead electrocardiography., Sci. Rep. 10 (2020) 20495. https://doi.org/10.1038/s41598-020-77599-6.

[22] Y.-Y. Jo, Y. Cho, S.Y. Lee, J. Kwon, K.-H. Kim, K.-H. Jeon, S. Cho, J. Park, B.-H. Oh, Explainable artificial intelligence to detect atrial fibrillation using electrocardiogram, Int. J. Cardiol. (2020). https://doi.org/10.1016/j.ijcard.2020.11.053.

[23] J. Kwon, Y. Cho, K.-H. Jeon, S. Cho, K.-H. Kim, S.D. Baek, S. Jeung, J. Park, B.-H. Oh, A deep learning algorithm to detect anaemia with ECGs: a retrospective, multicentre study, Lancet Digit. Heal. 2 (2020) e358-e367. https://doi.org/10.1016/S2589-7500(20)30108-4.

[24] J. Kwon, S.Y. Lee, K. Jeon, Y. Lee, K. Kim, J. Park, B. Oh, M. Lee, Deep LearningBased Algorithm for Detecting Aortic Stenosis Using Electrocardiography, J. Am. Heart Assoc. 9 (2020). https://doi.org/10.1161/JAHA.119.014717.

[25] J. myoung Kwon, K.H. Kim, J. Medina-Inojosa, K.H. Jeon, J. Park, B.H. Oh, Artificial intelligence for early prediction of pulmonary hypertension using electrocardiography, J. Hear. Lung Transplant. 39 (2020) 805-814. https://doi.org/10.1016/j.healun.2020.04.009.

[26] E.F. Schisterman, N.J. Perkins, A. Liu, H. Bondell, Optimal cut-point and its corresponding Youden index to discriminate individuals using pooled blood samples, Epidemiology. 16 (2005) 73-81. https://doi.org/10.1097/01.ede.0000147512.81966.ba.

[27] R.R. Selvaraju, M. Cogswell, A. Das, R. Vedantam, D. Parikh, D. Batra, Grad-CAM: Visual Explanations from Deep Networks via Gradient-Based Localization, in: Proc. IEEE Int. Conf. Comput. Vis., 2017: pp. 1;618-626. 
https://doi.org/10.1109/ICCV.2017.74.

[28] R.R. Selvaraju, M. Cogswell, A. Das, R. Vedantam, D. Parikh, D. Batra, Grad-CAM: Visual Explanations from Deep Networks via Gradient-Based Localization, Int. J. Comput. Vis. 128 (2020) 336-359. https://doi.org/10.1007/s11263-019-01228-7.

[29] Z. Zhang, M.W. Beck, D.A. Winkler, B. Huang, W. Sibanda, H. Goyal, Opening the black box of neural networks: methods for interpreting neural network models in clinical applications, Ann. Transl. Med. 6 (2018) 216-216. https://doi.org/10.21037/atm.2018.05.32.

[30] P.B. Soeters, R.R. Wolfe, A. Shenkin, Hypoalbuminemia: Pathogenesis and Clinical Significance, J. Parenter. Enter. Nutr. 43 (2019) 181-193. https://doi.org/10.1002/jpen.1451.

[31] Y. LeCun, Y. Bengio, G. Hinton, Deep learning, Nature. 521 (2015) 436-444. https://doi.org/10.1038/nature14539.

[32] J.G. Heaf, Albumin-Induced changes in the electrocardiographic QRS complex, Am. J. Cardiol. 55 (1985) 1530-1533. https://doi.org/10.1016/0002-9149(85)90967-1.

[33] J.E. Madias, Effect of changes in body weight and serum albumin levels on electrocardiographic QRS amplitudes, Am. J. Cardiol. 89 (2002) 1233-1235. https://doi.org/10.1016/S0002-9149(02)02315-9.

[34] L. Toma, A.M. Stanciu, A. Zgura, N. Bacalbasa, C. Diaconu, L. Iliescu, Electrocardiographic Changes in Liver Cirrhosis-Clues for Cirrhotic Cardiomyopathy, Medicina (B. Aires). 56 (2020) 68. https://doi.org/10.3390/medicina56020068. 
Table 1 Baseline characteristics

\begin{tabular}{|c|c|c|c|c|c|c|c|}
\hline \multirow[b]{2}{*}{ Characteristic } & \multicolumn{3}{|c|}{$\begin{array}{c}\text { Development and internal } \\
\text { validation dataset (Hospital A) } \\
\mathbf{n}=\mathbf{7 8 , 1 7 1}\end{array}$} & \multicolumn{3}{|c|}{$\begin{array}{c}\text { External validation dataset } \\
\text { (Hospital B) } \\
\mathbf{n}=\mathbf{3 7 , 1 9 0} \\
\end{array}$} & \multirow{2}{*}{$\begin{array}{l}p \\
\\
\quad p\end{array}$} \\
\hline & $\begin{array}{l}\text { Non- } \\
\text { hypoalbu } \\
\text { minemia }\end{array}$ & $\begin{array}{c}\text { Hypoalbu } \\
\text { minemia }\end{array}$ & $p$ & $\begin{array}{c}\text { Non- } \\
\text { hypoalbu } \\
\text { minemia }\end{array}$ & $\begin{array}{c}\text { Hypoalbu } \\
\text { minemia }\end{array}$ & $p$ & \\
\hline $\begin{array}{l}\text { Study population, } \mathrm{n} \\
(\%)\end{array}$ & 72,422 & 2,490 & & 36,099 & 1,322 & & 0.071 \\
\hline Age, yr, mean (SD) & $\begin{array}{l}58.81 \\
(17.20)\end{array}$ & $\begin{array}{l}72.61 \\
(14.68)\end{array}$ & $<0.001$ & $\begin{array}{l}53.16 \\
(17.22)\end{array}$ & $\begin{array}{l}71.74 \\
(14.77)\end{array}$ & $<0.001$ & $<0.001$ \\
\hline Male, n, (\%) & $\begin{array}{l}38,321 \\
(52.9)\end{array}$ & $\begin{array}{l}1247 \\
(50.1)\end{array}$ & 0.006 & $\begin{array}{l}17,642 \\
(48.9)\end{array}$ & $654(49.5)$ & 0.689 & $<0.001$ \\
\hline Heart rate, bpm $(\%)$ & $\begin{array}{l}72.55 \\
(18.60)\end{array}$ & $\begin{array}{l}87.92 \\
(24.79)\end{array}$ & $<0.001$ & $\begin{array}{l}72.05 \\
(16.62)\end{array}$ & $\begin{array}{l}87.38 \\
(22.58)\end{array}$ & $<0.001$ & 0.262 \\
\hline $\begin{array}{l}\text { PR interval, ms, } \\
\text { mean (SD) }\end{array}$ & $\begin{array}{l}170.89 \\
(30.14)\end{array}$ & $\begin{array}{l}169.47 \\
(36.68)\end{array}$ & 0.041 & $\begin{array}{l}166.01 \\
(26.55)\end{array}$ & $\begin{array}{l}165.00 \\
(32.53)\end{array}$ & 0.206 & $<0.001$ \\
\hline $\begin{array}{l}\text { QRS duration, ms, } \\
\text { mean (SD) }\end{array}$ & $\begin{array}{l}96.60 \\
(17.98)\end{array}$ & $\begin{array}{l}98.24 \\
(22.66)\end{array}$ & $<0.001$ & $\begin{array}{l}94.46 \\
(14.78)\end{array}$ & $\begin{array}{l}95.25 \\
(20.04)\end{array}$ & 0.060 & $<0.001$ \\
\hline $\begin{array}{l}\text { QT interval, ms, } \\
\text { mean (SD) }\end{array}$ & $\begin{array}{l}404.64 \\
(42.26)\end{array}$ & $\begin{array}{l}394.76 \\
(59.75)\end{array}$ & $<0.001$ & $\begin{array}{l}399.98 \\
(38.29)\end{array}$ & $\begin{array}{l}389.83 \\
(53.60)\end{array}$ & $<0.001$ & $<0.001$ \\
\hline QTc, ms, mean (SD) & $\begin{array}{l}437.63 \\
(33.69)\end{array}$ & $\begin{array}{l}465.31 \\
(43.17)\end{array}$ & $<0.001$ & $\begin{array}{l}432.24 \\
(31.07)\end{array}$ & $\begin{array}{l}460.25 \\
(38.60)\end{array}$ & $<0.001$ & $<0.001$ \\
\hline $\mathrm{P}$ axis, mean (SD) & $\begin{array}{l}43.49 \\
(30.10)\end{array}$ & $\begin{array}{l}44.49 \\
(38.42)\end{array}$ & 0.170 & $\begin{array}{l}44.30 \\
(27.47)\end{array}$ & $\begin{array}{l}42.47 \\
(32.18)\end{array}$ & 0.027 & $<0.001$ \\
\hline $\mathrm{R}$ axis, mean (SD) & $\begin{array}{l}39.51 \\
(44.47)\end{array}$ & $\begin{array}{l}35.31 \\
(55.51)\end{array}$ & $<0.001$ & $\begin{array}{l}41.52 \\
(39.16)\end{array}$ & $\begin{array}{l}29.88 \\
(48.38)\end{array}$ & $<0.001$ & $<0.001$ \\
\hline $\mathrm{T}$ axis, mean (SD) & $\begin{array}{l}44.77 \\
(47.94)\end{array}$ & $\begin{array}{l}66.89 \\
(74.53)\end{array}$ & $<0.001$ & $\begin{array}{l}38.81 \\
(35.91)\end{array}$ & $\begin{array}{l}56.87 \\
(63.75)\end{array}$ & $<0.001$ & $<0.001$ \\
\hline $\begin{array}{l}\text { Albumin, mean } \\
\text { (SD) }\end{array}$ & $4.48(0.36)$ & $\begin{array}{l}3.02 \\
(0.38)\end{array}$ & $<0.001$ & $4.30(0.30)$ & $\begin{array}{l}3.01 \\
(0.41)\end{array}$ & $<0.001$ & $<0.001$ \\
\hline
\end{tabular}


$1 \quad$ Figure legends

2 Figure 1 Performances of deep learning based model for detecting hypoalbuminemia

3 Legend: AUC denote area under the receiver operating characteristic curve, CI confidence

4 interval, DLM deep learning based model, ECG electrocardiography, NPV negative

5 predictive value, PPV positive predictive value, SEN sensitivity, and SPE specificity.

6 Figure 2 Sensitivity map of deep learning based model for detecting hypoalbuminemia

7 Legend: none

8 Figure 3 Cumulative hazard of developing hypoalbuminemia in patients with an

9 initially normal

10 Legend: none 


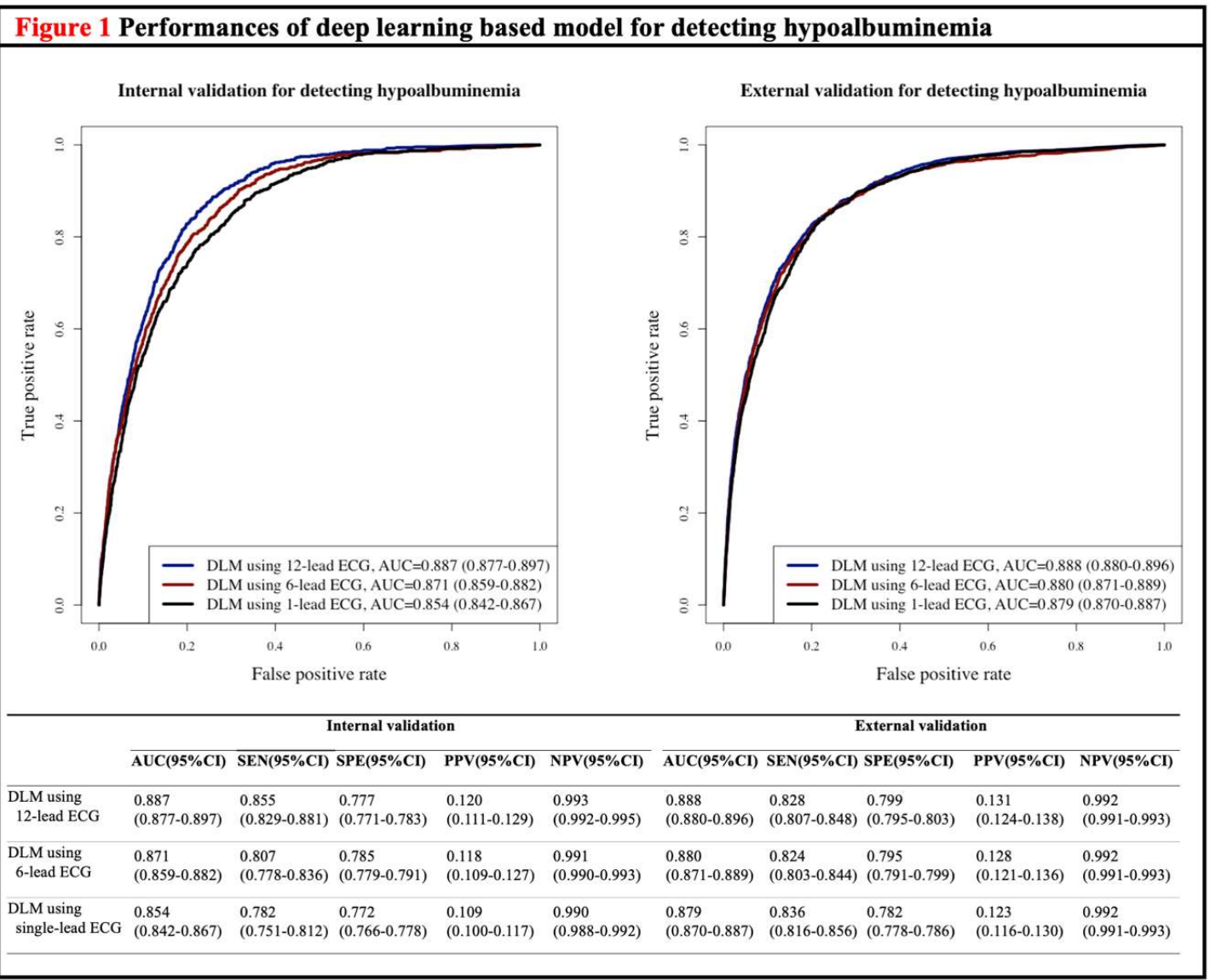

1 
Figure 2 Sensitivity map of deep learning based model for detecting hypoalbuminemia
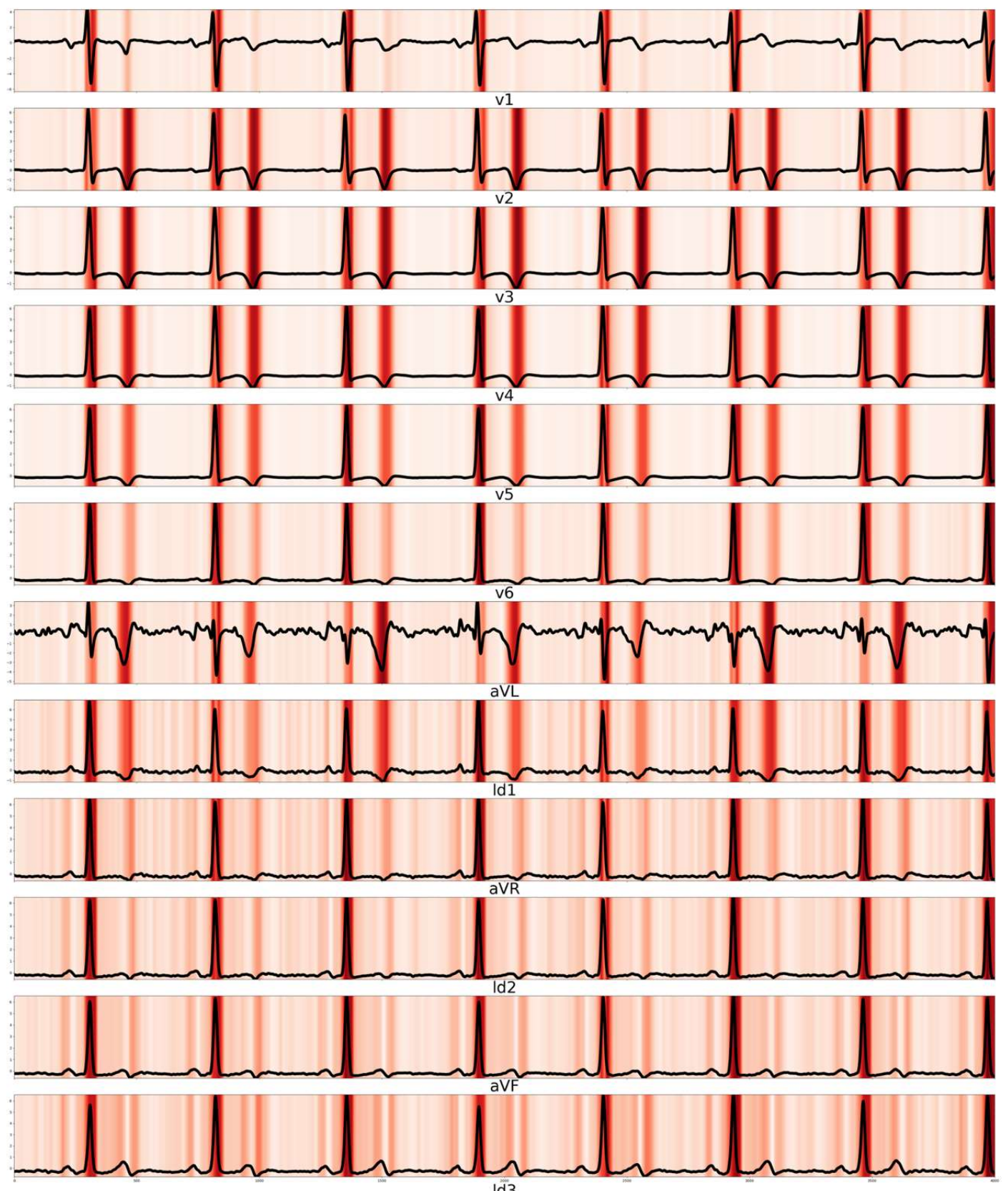


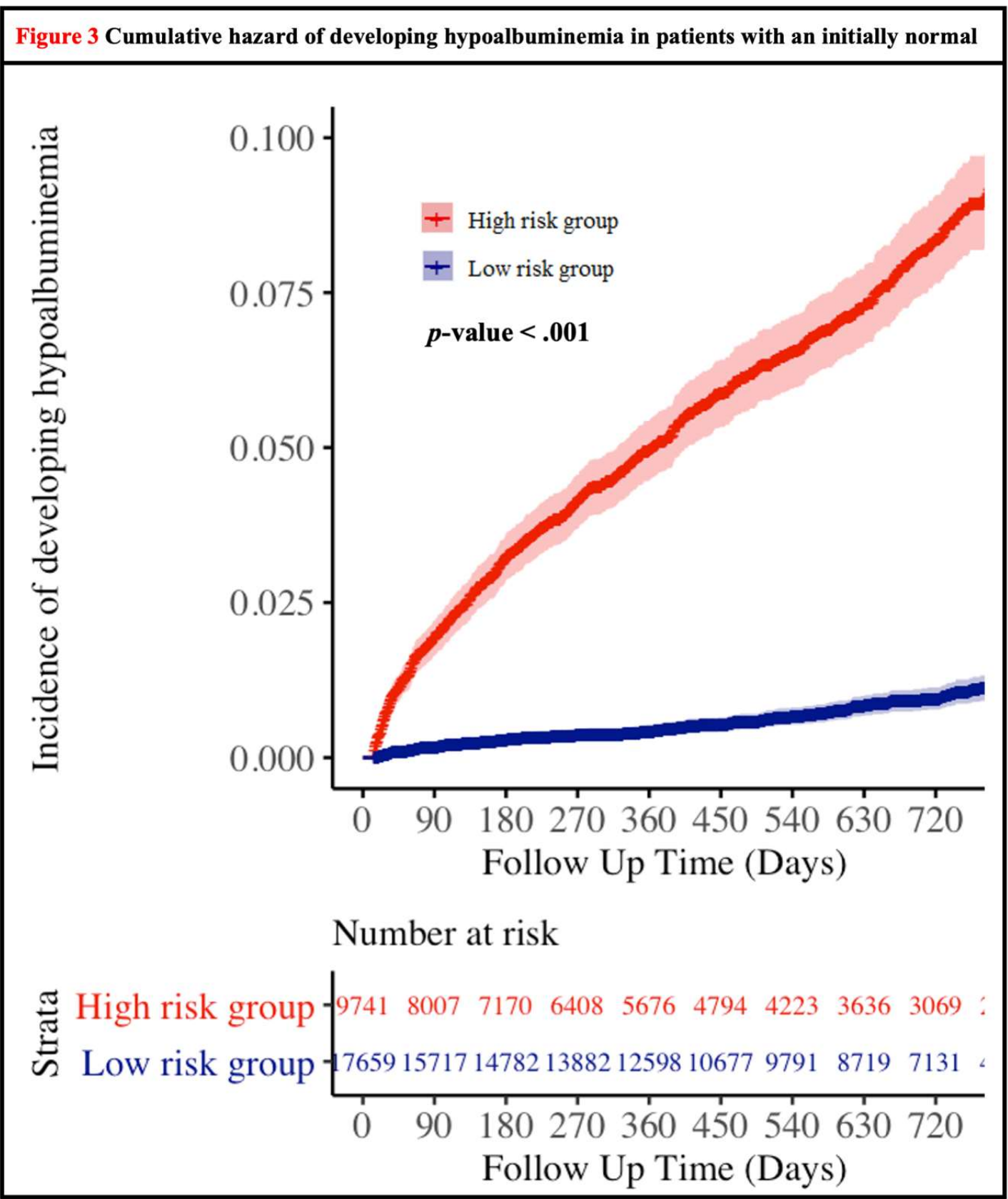

